\title{
Literature Review: Development and Effect Evaluation of Clean Development Mechanism (CDM)
}

\author{
Yang Lu \\ School of Economics and Management,Beijing Jiaotong University,BeiJing,China 100044 \\ ylu0321@163.com
}

Keywords: Clean Development Mechanism (CDM),Evaluation,China

\begin{abstract}
Clean Development Mechanism (CDM) is one mechanism that can bring significant economic and social benefits, can solve climate and environmental problems by market means, and it is also a win-win mechanism for cooperation between developed and developing countries. This paper will classify the related literature from the mode of development, international experience, legal issues, carbon financial, and CDM project evaluation.
\end{abstract}

\section{Introduction}

Clean Development Mechanism is one of the flexible performance mechanisms introduced in the Kyoto protocol. CDM allows Annex 1 parties together with non-Annex 1 parties to carry out GHG emission reduction project such as carbon dioxide. The emission reductions generated by those items may be made by the Annex 1 parties to discharge their commitments. For developed countries, CDM provides a flexible performance mechanism, but for developing countries, the CDM project can get some financial assistance and advanced technology.

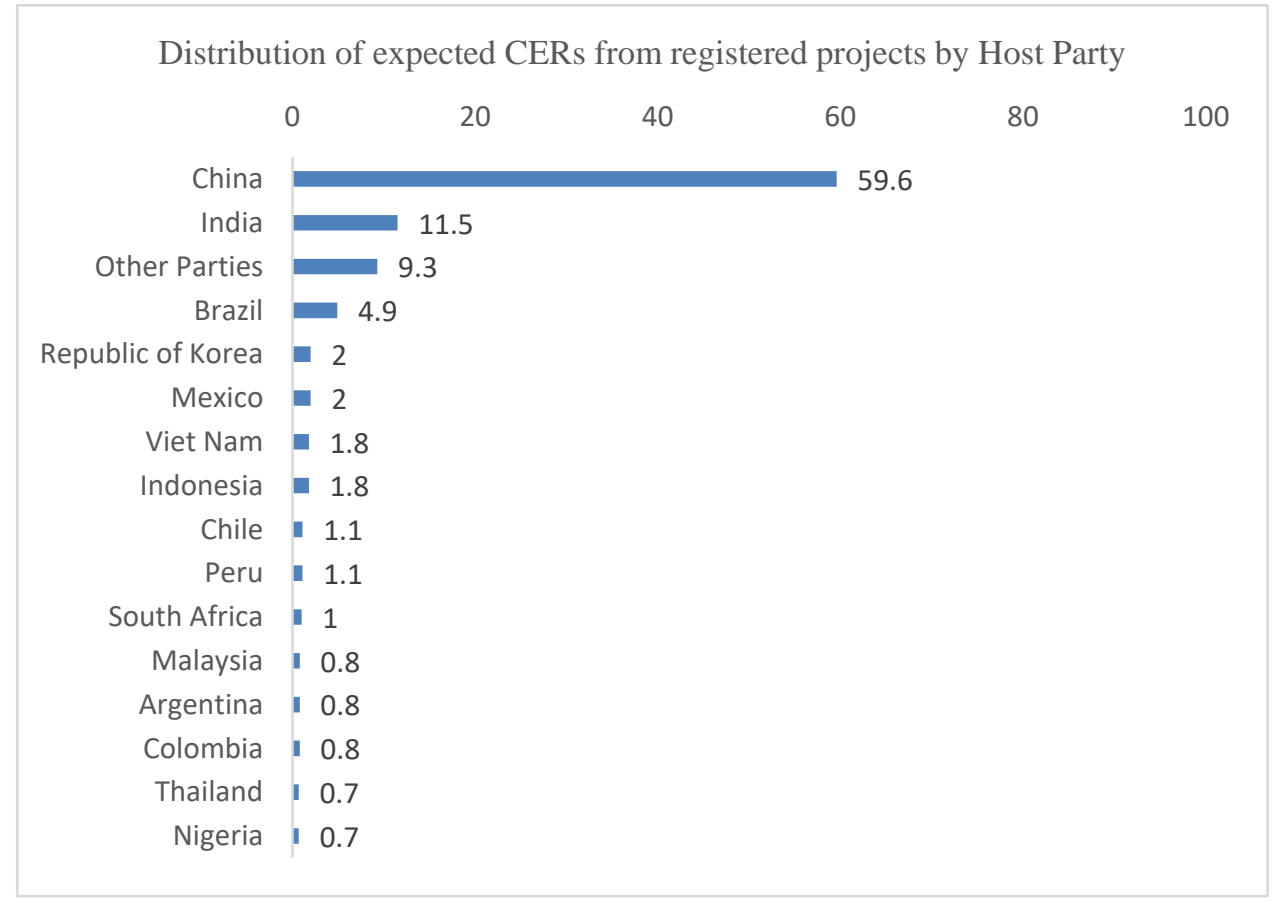

Figure 1. Distribution of expected CERs from registered projects by Host Party

At the end of 2002, the government of Holland and the China government signed the first contract of the CDM project in the Inner Mongolia Autonomous Region Huitengxile wind farm project, CDM officially entered in China. Until August 23, 2016, the National Development and Reform Commission approved all CDM projects 5074, as of July 14, 2015, all CDM projects registered in EB 3807. For the international market, the global CDM market is mainly among China, India and Brazil, these three countries accounted for nearly $80 \%$ of the total number of items, and China ranked first (Figure 1). The data showed that the CDM project has great potential in China. Developing clean 
energy mechanism, not only can promote the sustainable development of the world economy, but also can obtain technical and financial support through cooperation with developed countries.

\section{Review of research on Clean Development Mechanism}

At present, most of the literature is mainly focused on the CDM project itself, involving the types, problems, solutions and effect evaluation research. In its effect evaluation research, most of them construct index system from the sustainable development. On this basis, this paper summarises the relevant literature at home and abroad, and puts forward the possible shortcomings.

2.1. research on the development and problems of clean development mechanism. The implementation of Clean Development Mechanism (CDM) has brought great benefits to both developed and developing countries. In view of the present situation of Clean Development Mechanism, many scholars have carried on the thorough researches. They agreed that the CDM project has obtained the rapid development in our country. Shen Yali (2012) believes that China has become the largest supplier of CDM. China is recognised as the world's largest provider of greenhouse gas emission credits. This is because China has low labor costs, better environmental policies and economic development potential compared with developed countries, so the cost of emission reduction is low. This will not only bring huge economic benefits to China, but also promote the introduction and absorption of advanced technology.

China's CDM project development has both opportunities and challenges. On the one hand, China's CDM project developed rapidly, and will become the world's largest supplier; on the other hand, in the development process of CDM, it also exposed many problems.

Firstly, from the perspective of CDM project development, the type of project structure is single and the regional distribution is unbalanced. Patrick Bayer (2013) found that high power consumption through an analysis of 2097 CDM projects in China 30 province from 2004 to 2009, low per capita income, lack of foreign direct investment are conducive to the implementation of CDM projects, the power consumption and the implementation of foreign direct investment influence CDM in particular. You Daming, Liu Yun Xi (2016) in order to study the regional differences of CDM project distribution in China, first use factor analysis to extract the regional characteristics index, and then cluster the method to divide China's provinces into 4 types of regional. Then studying the influence factors of 2005 - 2013 years of regional CDM distribution project. The results showed that investment has inhibitory effect on the CDM project of regional distribution technology, technical level is high and its influence area is bigger; the level of economic development is positively correlated with the distribution of CDM projects, the region can bring more CDM investment opportunities by enhancing economic strength and investment because of its energy and greenhouse gas emissions are closely related, so the CDM project distribution has positive effect. The industrial structure is the main factor causing the regional distribution differences of CDM projects. The proportion of the second industries in the regional industrial structure has a positive impact on the distribution of CDM projects. In the international market, there is also the phenomenon of uneven distribution. Martina Jung (2005) used cluster analysis that was conducted according to the appeal of the clean development mechanism of the host country. The results showed that only a small part of the potential host country will attract investment in clean development mechanism, size is as follows: Chinese, India, Brazil, Argentina, Mexico, South Africa, Indonesia, Thailand.

Secondly, as for the enterprise level, high-level technology transfer has not reflected in the development of CDM. The Dechezleprtre (2008) Using 644 registered CDM projects as of May 2007, focusing on the transfer of greenhouse gas mitigation technologies resulting from CDM. From a descriptive point of view, the data shows that technology transfer occurs in more than $40 \%$ CDM projects. Kasturi Das (2011) presents a definition of the scope of technology transfer for clean development mechanism through research. This definition applies to 1000 registered CDM projects. The study found that CDM projects involve little core technology transfer. From 1000 research projects, only about 265 involved in technology transfer. 
Finally, for the government level, the relevant policy guidance is not clear, carbon trading market system is not perfect, which made our country in the inferior position in the international carbon emissions trading market. Gong Wei (2011) believes that the clean development mechanism has a geographical distribution, project types, technology transfer and other issues in promoting the sustainable development to developing countries, there is no legal rules on the definition of sustainable development without the international standard of the defects and shortcomings, which needs to improve. Zhang Yaxin, Li Tao, (2013) believes that the current international CDM project competition is more intense, but China's CDM project in the international financing process has always been at a disadvantage. The development of China's inter regional CDM investment and financing model is conducive to reduce the dependence of foreign domestic CDM projects, alleviate the shortage of share of emissions rights, and promote the development of domestic CDM projects.

2.2. research on the effect evaluation of clean development mechanism. At present, there are few literatures on the evaluation of CDM effect at home or abroad, and these literatures mostly are using index system to evaluate sustainable development. Through the overall project research, we can better reflect the effect of the implementation of clean energy mechanism, to provide appropriate theoretical basis for the development of relevant policies.

Zhang Shuwei, Liu Deshun sustainability evaluation indexes of multi-objective decision theory system based on the CDM project, Inner Mongolia Huitengxile wind power CDM project was evaluated. The author divides sustainable indicators into social development, economic development and environmental development as second indicator, and finds out a number of indicators under the level of second indicator, a total of twelve indicators. By the reference line project as a reference, to determine the score and weight of each attribute, you can determine the overall score according to the utility theory,and then determine the reference line CDM project contribution to sustainable development and contribution. Feng Xiangzhao, Li Liping (2010) took multi-attribute utility theory (MATA - CDM) as a guidance, the evaluation index system of sustainable development is constructed including four levels: target layer, system layer, variable layer and factor layer, calculate the loudness of the sustainable development of the CDM project. Chen Kun (2009) took the CDM project and baseline project comparison as the basic method of constructing model, establish the evaluation matrix to the multi-objective decision theory, using AHP to establish the index system, using the unified calculation processing and the utility value of the utility function of the index. Yongfu Huang, Terry Barker analyzed the long-term effects during 1993-2009 qualified 80 CDM project on carbon dioxide emission reduction according to the utility theory. Through the analysis of CDM projects in many countries, it is confirmed that the decline of $\mathrm{CO} 2$ emissions is related to CDM projects. Help developing countries to effectively develop CDM projects to achieve low-carbon development. Karen Holm Olsen submitted the designed document based on the text of 744 project for reviewing before March 2006 sustainability evaluation classification. The results of this study show that: methane emission reduction projects have a higher environmental protection, renewable energy projects with high socio-economic status. Small scale projects contribute an average of slightly higher than large projects, and have high socio-economic benefits, while large projects contribute to relatively more air quality, water, health and other benefits.

\section{Conclusion}

This article mainly about the CDM project development and the CDM project effect influence two aspects combs. From the research content, because of China's strong resource endowment, CDM projects in China developed rapidly, improved local environmental conditions, also brought technical support and financial assistance. But there are also regional distribution imbalance, such as the core technology transfer less, the legal system is not perfect, carbon trading market imperfections. To solve these problems, scholars also give relevant recommendations from two levels of enterprise and government. From the point of view of research methods, the evaluation of CDM project is mainly from the construction of index system, and many studies are on the impact of sustainable economic development evaluation. 
As one of the three mechanisms of the Kyoto Protocol, CDM provides conditions for developed countries as well as developing countries in order to achieve "win-win" in reducing greenhouse gases, which has great theoretical value. In recent years, China has made important contributions to the realization of quantified emission reduction targets and the responsibility to global climate change by vigorously developing CDM projects.

\section{References}

[1] You Daming, Liu Yunxi. Regional difference of CDM project distribution in China [J]. Ecological Economics, 2016, 32 (5): 95-101.

[2] Bayer P, Urpelainen J, Wallace J. Who uses the Clean Development Mechanism? An empirical analysis of projects in Chinese provinces [J]. Global Environmental Change, 2013, 23(2):512521.

[3] Martina Jung. Host country attractiveness for CDM non-sink projects[J]. Energy Policy, 2005, 34(15):2173-2184.

[4] Kasturi Das. Technology Transfer Under the Clean Development Mechanism: An Empirical Study of 1000CDMProjects[J]. Ssrn Electronic Journal, 2011.

[5] Gong Wei. On the clean development mechanism (CDM) defects and perfection of the goal of sustainable development in international climate change legislation from the perspective of political and legal rules of [J]. Energy Policy ,2011 (9): 130-136.

[6] Zhang Yaxin, Li Tao, and. Discussion on CDM investment and financing model in China [J]. Contemporary Economic Research, 2013 (5): 51-56.

[7] Feng Xiangzhao. Evaluation of impact of China CDM project on sustainable development [J]. Population and Resources and Environment of China, 2010, 20 (7): 129-135. 